\title{
Termination of Electrical Storm After Double Valve Replacement Using Sympathectomy
}

\author{
Nael Al-Sarraf, FRCSC, ${ }^{1}$ Derar S. AlShehab, FRCSC, ${ }^{1}$ Essa M. AlGhunaim, MD, ${ }^{1}$ \\ Mohamed F. Elkhalifa, MRCP, ${ }^{2}$ Archana Matthias, MD, ${ }^{1}{ }^{2}$ amal Al-Fadhli, FRCSC ${ }^{1}$ \\ ${ }^{1}$ Department of Cardiothoracic Surgery, Chest Diseases Hospital, Kuwait City, Kuwait; ${ }^{2}$ Department of Cardiology, \\ Chest Diseases Hospital, Kuwait City, Kuwait
}

\section{ABSTRACT}

Electrical storm is a fatal condition unless aborted. Various treatments are available, each with its own limitations. Here, we present a case in which all forms of therapy failed except sympathectomy, which terminated the storm successfully.

\section{INTRODUCTION}

Electrical storm is characterized by multiple episodes of ventricular tachycardia or fibrillation (VT or VF) in a short period of time. It manifests as 3 or more shocks delivered within a 24-hour period [Vaseghi 2014]. Sympathectomy has been used successfully in cases of refractory ventricular arrhythmias (VT or VF) complicating coronary spasm, ischemic cardiomyopathy [Cardona-Giarache 2017], prolonged QT syndrome [Schwartz 2004], arrhythmogenic right ventricular cardiomyopathy [Ajijola 2012a], and catecholaminergic polymorphic V'T [Wilde 2008]. However, its use in aborting electrical storm after double valve replacement has never been reported.

\section{CASE REPORT}

A 31-year-old previously healthy man presented with fever and dyspnea. He was in New York Heart Association class IV dyspnea and required intubation on admission. He had 1 episode of VT that required direct current (DC) cardioversion and was extubated 2 days later. His blood cultures were negative for any microorganisms. Echocardiography showed severe aortic regurgitation with mobile vegetation attached to noncoronary and left coronary cusps (NCC and LCC). Ejection fraction was $45 \%$ with dilated left ventricle and global hypokinesia and moderate to severe eccentric mitral regurgitation. There was a suspicion of aortic root abscess.

The patient had persistent fever despite being on intravenous antibiotics and was transferred to our center for urgent surgery. He underwent mechanical aortic and mitral valve

Received October 17, 2019; accepted December 9, 2019.

Correspondence: Dr. Nael Al-Sarraf, Al-fabriah, PO Box 1134, Postal Code 46312, Kuwait; 96598882921 (e-mail: trinityq8@hotmail.com). replacements with closure of aortic root fistula using a bovine pericardial patch. Both coronary artery ostia were seen and protected. He was weaned off cardiopulmonary bypass on small doses of levosimendan and norepinephrine.

The patient was extubated 12 hours after surgery and remained in sinus rhythm. He was neurologically intact and maintained good urine output. All inotropic support was stopped on day 1 . His lactate was normal, and he was afebrile.

On postoperative day 3, the patient developed recurrent VT (Figures $1 \mathrm{~A}$ and $1 \mathrm{~B}$ ). He had 4 to 5 episodes that required DC cardioversion and reintubation with deep sedation. The cause of the recurrent VT was unclear; it was believed to be due to irritable myocardium and a sequalae of sepsis causing a hyperadrenergic stimulation. At that time, he was on epinephrine $0.05 \mu \mathrm{g} / \mathrm{kg} / \mathrm{min}$ and norepinephrine at 0.15 to 0.2 $\mu \mathrm{g} / \mathrm{kg} / \mathrm{min}$ with lignocaine and amiodarone infusion. Serum concentrations of potassium and magnesium were 4.2 and 1.2 $\mathrm{mmol} / \mathrm{L}$, respectively, at the time of $\mathrm{VT}$ storm. Infusion of lignocaine and amiodarone were started, and the doses were optimized. Magnesium sulfate was also administered intravenously. Despite this, his VT recurred.

Coronary angiography was performed and was normal, and echo showed well-functioning valves. Fast pacing was tried with no success. Ventricular ablation was deferred in view of mechanical mitral valve. Beta blockers were started but were not successful. Norepinephrine was switched over to vasopressin to avoid beta-agonist effects on the heart, and epinephrine was weaned to the lowest feasible dose. Nonetheless, the patient continued to have multiple VTs and required multiple DC cardioversions (3 to 8 times a day).

At that point, we had no option but to proceed with uniportal bilateral videoscopic sympathectomy. The patient was in semi-sitting position at $45^{\circ}$ under double lumen endotracheal intubation, and the contralateral lung was selectively ventilated on each side of sympathectomy. The left side approach was performed first, then the right side. One incision (1 cm long) in the anterior axillary line at the fourth intercostal space was performed. A small wound protector was placed, followed by the introduction of a $5-\mathrm{mm}$ telescope. The sympathetic chain was identified and dissected out from its bed from the lower half of stellate ganglion and T2 to T4 (Figure 2). The sympathetic nerve was then resected and extracted. The nerve of Kuntz was also cauterized at T2 level across the rib. Chest drains were inserted, 1 


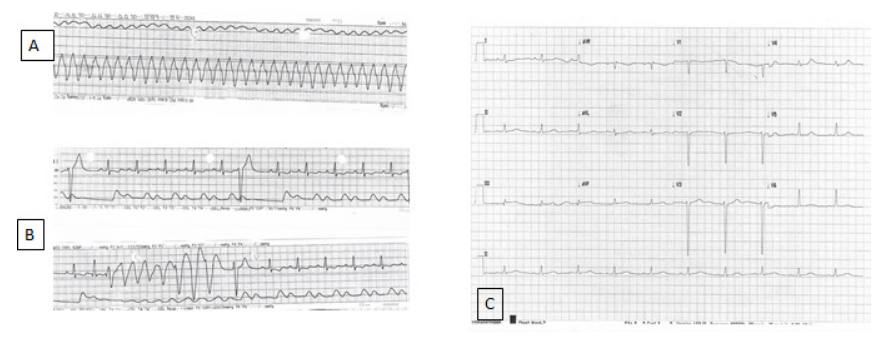

Figure 1. A, Rhythm strip showing ventricular tachycardia. B, Rhythm strip showing premature ventricular contraction with a run of ventricular tachycardia. C, Electrocardiogram showing sinus rhythm on discharge.

on each side, and removed 24 hours later. All samples were verified histologically.

After the sympathectomy, the patient had no V'T attacks and was in sinus rhythm with a heart rate of $64 \mathrm{bpm}$. Serum potassium and magnesium were 4.3 and $1.1 \mathrm{mmol} / \mathrm{L}$, respectively. All inotropic and vasopressor supports were stopped, and metoprolol dose was adjusted to $50 \mathrm{mg}$ twice a day.

The patient was extubated 1 day after sympathectomy. His kidney and liver function improved. He was kept under close observation and became fully ambulatory. There was no instability in condition and no dysrhythmias.

On day 6 after the sympathectomy, he was transferred to the ward on continuous telemetry monitoring. During the 6-week hospital stay for intravenous antibiotic therapy, VT did not recur. All subsequent electrocardiograms showed sinus bradycardia (Figure 1C). Predischarge echo showed well-functioning valves with no paravalvular leak, and the ejection fraction returned to normal (65\%). The patient was evaluated by the electrophysiology team; in the absence of recurrent episodes of VT, no implantable cardioverter-defibrillator (ICD) was inserted. He was discharged home on Coumadin, aspirin, and metoprolol.

The patient was followed up in the clinic in the next 3 months, and he was back at work and had no issues. He remains in sinus rhythm persistently. Informed consent was obtained from the patient for the publication of this report and the data accompanying it.

\section{DISCUSSION}

Various therapies have been used to treat ventricular arrhythmias, including pharmacologic interventions, stellate ganglion blockade, thoracic epidural injections, electrical stimulation of the vagus nerve, and spinal cord elements via implantable devices and surgical cardiac sympathetic denervation [Ajijola 2012b]. Percutaneous stellate ganglion block has a temporarily effect and is usually followed by surgical sympathectomy; it is reserved for patients who cannot tolerate surgical sympathectomy.

In contrast, surgical sympathectomy is a permanent procedure providing permanent sympathetic block and long-term suppression of ventricular arrhythmias [Cardona-Guarache 2017]. The videoscopic approach improves access to the
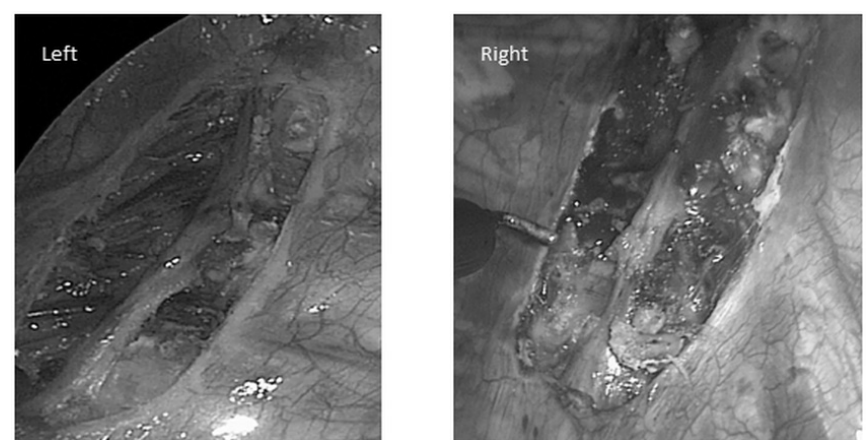

Figure 2. Intraoperative images for both left and right sympathetic chains dissected from their beds.

lower aspect of the thoracic sympathetic chain and is a minimally invasive procedure. It carries excellent results. The main side effects are hemothorax, pneumothorax, Horner's syndrome, altered temperature sensation, and sweating of the face and upper extremities [Vaseghi 2014; Ajijola 2012b]. In a recent paper, in a series of 64 patients with catecholaminergic polymorphic VT, the cumulative event-free survival at 2 years was $81 \%$ with left cardiac sympathectomy [De Ferrari 2015].

The rationale of this procedure is that an increase in sympathetic tone promotes arrhythmia and that the lower cervical and upper thoracic sympathetic ganglia help regulate cardiac sympathetic innervation. However, there is a functional asymmetry in the innervation of the heart, in that the left sympathetic nerves affect the VF threshold of the left and posterior walls of the heart, and the right sympathetic nerves influence the heart rate and arrhythmia vulnerability of anterior aspect of the heart [Cardona-Guarache 2017].

A variety of reasons may account for inefficiencies of left cardiac sympathetic denervation alone. Because of anatomic variability in the course of preganglionic sympathetic fibers, resection of the lower half of the stellate ganglion and the ganglia of T2-T4 may not sever all the sympathetic connections to the heart. Preganglionic sympathetic fibers may extend to C7 and T5 ganglia and may assume the role of the previously resected sympathetic ganglia. In addition, the right cardiac sympathetic chain may hypertrophy and extend nerve sprouts to the region supplied by the resected left side ganglia [Ajijola 2012b]. In addition, right sympathetic denervation has been shown to add extra benefit, and the rate of success is reported to be higher when bilateral sympathectomy is performed [Cardona-Guarache 2017; Ajijola 2012a]. Although a literature review showed successful termination of VT storm in coronary spasm, ischemic cardiomyopathy [CardonaGuarache 2017], prolonged QT syndrome [Schwartz 2004], arrhythmogenic right ventricular cardiomyopathy [Ajijola 2012a], and catecholaminergic polymorphic VT [Wilde 2008], its use in the cardiac surgery setting has been reported only in the context of remote ischemic cardiopathy after coronary artery bypass graft. Factors that seem to influence its success are the extent of sympathectomy performed, bilateral versus unilateral sympathectomy, and the underlying condition causing the V'T storm. 


\section{CONCLUSION}

We report a case of life-threatening electrical storm that was successfully aborted by bilateral videoscopic sympathectomy, obviating the need for ICD implantation in a patient who underwent double valve replacement for native valve endocarditis. The use of such a procedure may have a role in terminating such fatal arrhythmias.

\section{REFERENCES}

Ajijola OA, Lellouche N, Bourke T, et al. 2012. Bilateral cardiac sympathetic denervation for the management of electrical storm. J Am Coll Cardiol 59:91-92.

Ajijola OA, Vaseghi M, Mahajan A, Shivkumar K. 2012. Bilateral cardiac sympathetic denervation: Why, who and when? Expert Rev Cardiovasc Ther 10:947-949.
Cardona-Guarache R, Padala SK, Velazco-Davila L, et al. 2017. Stellate ganglion blockade and bilateral cardiac sympathetic denervation in patients with life-threatening ventricular arrhythmias. J Cardiovasc Electrophysiol 28:903-908.

De Ferrari GM, Dusi V, Spazzolini C, et al. 2015. Clinical management of catecholaminergic polymorphic ventricular tachycardia: The role of left cardiac sympathetic denervation. Circulation 131:2185-2193.

Schwartz PJ, Priori SG, Cerrone M, et al. 2004. Left cardiac sympathetic denervation in the management of high-risk patients affected by the long-QT syndrome. Circulation 109:1826-1833.

Vaseghi M, Gima J, Kanaan C, et al. 2014. Cardiac sympathetic denervation in patients with refractory ventricular arrhythmias or electrical storm: Intermediate and long-term follow-up. Heart Rhythm 11:360-366.

Wilde AA, Bhuiyan ZA, Crotti L, et al. 2008. Left cardiac sympathetic denervation for catecholaminergic polymorphic ventricular tachycardia. N Engl J Med 358:2024-2029. 